\title{
De l'usage expérimental des archives audio dans la recherche sur la mémoire des spectateurs
}

On the Experimental Use of Audio Archives for Researching Spectator Memory

\section{Hélène Bouvier}

\section{(2) OpenEdition}

\section{Journals}

Édition électronique

URL : https://journals.openedition.org/rsl/1743

DOI : $10.4000 /$ rsl. 1743

ISSN : 2271-6246

Éditeur

Éditions Rue d'Ulm

Référence électronique

Hélène Bouvier, «De l'usage expérimental des archives audio dans la recherche sur la mémoire des spectateurs », Revue Sciences/Lettres [En ligne], 6 | 2019, mis en ligne le 10 décembre 2018, consulté le 30 juillet 2021. URL : http://journals.openedition.org/rsl/1743; DOl : https://doi.org/10.4000/rsl.1743

Ce document a été généré automatiquement le 30 juillet 2021

(c) Revue Sciences/Lettres 


\section{De l'usage expérimental des archives audio dans la recherche sur la mémoire des spectateurs}

On the Experimental Use of Audio Archives for Researching Spectator Memory

Hélène Bouvier

1 Ces réflexions sur l'usage des archives audio découlent d'une situation de recherche à plusieurs titres particulière. Son objet, la mémoire théâtrale des spectateurs, et autant que possible leur mémoire sonore, déplace le foyer plus couramment centré sur les productions ou les acteurs directs de la création théâtrale, vers leur destinataire ultime, le spectateur, et de manière rétrospective. En effet, la période étudiée par l'ANR ECHO dans laquelle cette recherche s'inscrit, la seconde moitié du xxe siècle, exclut de facto les enquêtes à la sortie de spectacles contemporains.

2 L'archive audio ${ }^{1}$ ne sert donc pas ici à l'étude directe de son contenu mais remplit des usages très différents les uns des autres: elle soutient l'effort de compréhension de l'effet et de la trace qu'un spectacle a pu produire sur des publics passés, et elle contribue à la formation historique et auditive du chercheur pour son travail d'enquête et d'entretien. Elle pourra aussi nourrir l'écoute rétrospective du spectateur-ancien témoin dans une étape ultérieure. Enfin, le simple fait d'enregistrer ces entretiens produit à son tour des documents audio, de même statut que ceux d'un terrain ethnographique, recueillis de première main et travaillés par le chercheur qui en maitrise toute la chaîne de production, le contexte matériel et humain, et les choix opérés à leur source, à la différence des archives théâtrales audio des fonds référencés, dont une part importante de l'élaboration reste inaccessible au chercheur, en tant que source secondaire non collectée directement par lui, ouvrant des champs d'interrogations presque insondables.

3 Tel est le feuilletage archivistique que je vais tenter de déplier succinctement. Je présenterai d'abord le cadre du questionnement, puis le processus de consultation des 
archives audio, enfin l'impact de leur écoute pour le chercheur, l'objet d'étude qu'est la mémoire sonore du théâtre, et potentiellement le spectateur lui-même.

\section{Le cadre du questionnement}

Il est parti de deux postulats :

- Un entretien est un échange et un partage, conditionné à sa mise en mots, dont l'objet scientifique est autant tributaire de l'interaction qui s'y déroule que des matériaux qui y sont débattus, exposés, écoutés ${ }^{2}$.

- La mémoire théâtrale concerne des expériences vécues, par définition très personnelles, et non pas une connaissance abstraite ou une élaboration strictement intellectuelle de l'objet « théâtre » ou même d'objets discrets que seraient les « représentations théâtrales ${ }^{3}$ ».

5 À ces hypothèses se sont aussitôt agrégées deux questions :

- Dans ces conditions, en recourant aux archives audio dans sa préparation, le chercheur favorise-t-il sa rencontre avec un spectateur de théâtre?

- Quel est l'intérêt particulier des archives audio du théâtre, parmi les autres sources disponibles, écrites et visuelles, pour la compréhension et l'analyse de la mémoire théâtrale d'autrui?

6 Sur le plan pratique, les options ouvertes pour l'écoute des archives audio qui se sont dégagées au cours de la recherche, autant pour le chercheur que pour le spectateur sollicité, correspondent aux questions suivantes :

- Écouter quand : plutôt avant ou plutôt après un entretien?

-Écouter quoi : des spectacles entiers ou des extraits, enregistrés en public ou en studio?

-Écouter comment : avec ou sans support écrit ou visuel, avec ou sans prise de notes?

\section{Les étapes de la consultation des archives audio}

7 Dès le début de cette recherche et avant de commencer les entretiens, j'ai voulu écouter une pièce en entier et enregistrée en public. Mon but était alors de plonger dans une époque révolue et qui m'était inconnue, une pièce que je n'avais pas lue ni vue, des acteurs dont je n'avais jamais entendu la voix, une relation troupe/public que je n'avais jamais entendue. Je concevais aussi cette écoute comme l'installation d'un possible filigrane de référence personnelle pour les entretiens à venir. Ce sont les archives audio de la BnF qui ont permis cette étape véritablement initiatrice.

En commençant avec l'enregistrement public du 24 janvier 1957 de Mère Courage, de Bertolt Brecht, mise en scène par Jean Vilar au TNP à Chaillot, l'archive audio m'a "sauté aux oreilles ", révélant plusieurs éléments frappants ${ }^{4}$ :

- les réactions du public, l'état aural de la salle : toux, rires aux répliques, aux mots d'humour, au ridicule de certains personnages (comme l'Aumônier), rires de bon cœur, applaudissements même au cours d'une scène, bruits dans la salle, bruit et brouhaha de sortie de salle ;

- la diction de la comédienne Germaine Montero dans le rôle de Mère Courage ;

- l'importance de la musique (tambour, clairon, trompettes, accordéon, piano, flûte), le bruit des déplacements sur scène de l'orchestre, des pas, cloches, coups de feu, canonnades. 
Cette première expérience d'écoute a très bien rempli sa fonction de vecteur, même imparfait, vers une époque révolue, et d'une manière plus profonde et plus efficace que la relation écrite ou photographique. J'ai eu l'impression, ce faisant, qu'elle avait ouvert un chemin ou plutôt déclenché un " accord », comme on accorde des instruments entre eux, dans les échanges avec les interlocuteurs se remémorant le spectacle, ou même se référant plus globalement à cette période, ce lieu, cette troupe. Les personnes les plus âgées, qui ont vu cette pièce, en ont gardé un souvenir marquant, lié d'abord à Germaine Montero, son jeu et sa voix, et aux chansons et à la musique parues ensuite en disque, mais aussi à la découverte de Brecht, à la violence du message, au moment (enfance, adolescence ou jeune adulte) où ce choc s'est produit dans leur vie ${ }^{5}$.

10 Peu familière avec le répertoire de théâtre sollicité, j'ai ensuite continué ce que je faisais dans un autre contexte culturel et pour d'autres formes de théâtre ${ }^{6}$, avec le même souci de me rapprocher à la fois de l'objet d'étude (la période historique et les représentations théâtrales) et des protagonistes (les amateurs et professionnels de théâtre). Pour me préparer aux entretiens par rapport au cadre défini par l'ANR ECHO, mais aussi en fonction des indices reçus lors de la première prise de contact, par téléphone ou par mail, j'ai donc commencé par consulter toutes sortes de documents papier et d'archives sonores (extraits audio et filmés en ligne), comme pour ouvrir un canal d'écoute avec mon futur interlocuteur, quels que soient les spectacles, les lieux ou les dates évoqués spontanément.

11 Pourtant, dès les premiers entretiens, la spécificité du sujet - des mémoires personnelles portant sur une longue période et recueillies oralement par autrui - a relativisé ce premier élan documentaire. J'ai constaté que mon écoute de la personne interviewée ne gagnait pas forcément en acuité en accumulant de mon côté plus de références objectivement communes, et qu'elle pouvait même perdre en qualité. J'y reviendrai plus loin.

12 Après une vingtaine d'entretiens, j'ai ressenti à nouveau le besoin de retourner aux archives de la BnF, cette fois plus comme un guide vers l'analyse qui s'amorçait que comme un soutien pour les entretiens futurs. Quels que soient leurs goûts ou leurs parcours, mes interlocuteurs qui avaient connu «la période Vilar» au TNP ou en Avignon s'y référaient en un bloc cohérent et sensible; j'ai donc repris l'écoute des archives à la BnF par ce répertoire. Les bribes de spectacles glanées dans les entretiens m'ont fait revenir sur mon premier dédain pour les collections d'extraits sonores : ces florilèges correspondent aussi à nos mémoires trouées comme des dentelles mais gardent comme elles leur pouvoir d'émotion au fil des ans. En fait, l'exhaustivité ne fait pas bon ménage avec le travail de remémoration.

13 L'écoute des archives audio a donc repris avec Les Grandes Heures du TNP, 1969: deux disques d'extraits de vingt-quatre spectacles donnés entre 1951 et 19637. Très informative, conçue comme un exemplier de la période Vilar au TNP, la succession des extraits provoque une audition particulière, qui oriente d'emblée vers la comparaison et l'analyse, et la notice indiquant la distribution permet de fixer l'écoute des voix. Pratiquement toutes les plages contiennent de la musique ou une chanson, et l'adéquation de la musique à la tonalité de la scène est surprenante. J'ai aussi été frappée par la qualité des dictions, qu'elles soient sur scène en public ou au micro en studio ; la richesse des textes qui n'entravent pas l'adhésion et la concentration d'un public manifestement familial et pas forcément érudit ; le mélange des générations et leurs manifestations vocales. 
papier (programmes, catalogues, photographies) ont été rapidement consultés, en accompagnement simultané ou décalé9. J'ai écouté de cette manière une représentation de Lorenzaccio, de Musset, enregistrée en public, en $1954^{10}$, d'où ressortent les voix de Gérard Philipe, Daniel Ivernel, Jean Vilar, Michel Vitold, et la présence active du public. L'écoute de La Tragédie du roi Richard II, de Shakespeare, enregistrée en public la même année ${ }^{11}$, révèle particulièrement bien le travail musical de Maurice Jarre, avec les réactions et une présence du public très audibles. La consultation des quelques photos $\mathrm{du}$ programme et de la pièce dans le programme TNP, avant son écoute audio, m'a d'ailleurs paru largement suffisante pour jouir de la représentation audio «presque comme un spectateur dans la salle». Il m'a semblé que cet assemblage inégal d'éléments visuels et de guide de scénario, laissant finalement la primauté à l'ouïe, a même favorisé l'écoute des voix, du jeu et du public, pour un enregistrement qui se déroule pourtant sur trois heures. La dernière pièce écoutée en entier est L'Avare de Molière, enregistrée en 1956 devant un public à l'enthousiasme contagieux, une salle, enfants et adultes mêlés, acquise dès le début, et qui acclame et ovationne « sa » troupe à la fin après avoir réagi tout au long de la représentation ${ }^{12}$.

\section{Quel est l'impact de l'écoute des archives audio dans cette recherche fondée sur des entretiens?}

16 Toutes les options d'écoute des archives audio évoquées au début de cette présentation ont été en définitive pratiquées à des moments différents, et soulèvent des questions sur chaque étape de cette recherche sur la mémoire des spectateurs.

17 À commencer par la relation entre le chercheur et son interlocuteur lors de l'entretien, fondée sur la confiance et la curiosité mutuelles. Il est logique de penser que l'écoute audio avant un entretien permet au chercheur d'approcher des références de son interlocuteur par cette expérience sensible, et donc d'augmenter sa compréhension ou sa connivence. Pourtant, que ce soit pour Mère Courage, le seul spectacle écouté en entier avant tous les entretiens, ou bien les tout petits extraits saisis en ligne, en écho aux premières prises de contact, cette pratique ne me paraît pas déterminante. La qualité d'écoute pendant l'entretien est à mon avis un facteur beaucoup plus important. Ainsi, la connaissance aiguisée d'une pièce, d'une mise en scène ou même d'une représentation donnée, peut conduire à une attitude directive, interventionniste, qui peut être contre-productive dans cette quête d'une mémoire fragile et incomplète, ou encore pire, qui peut mettre en défaut l'interlocuteur par la précision de détails oubliés ou simplement non pertinents pour lui. La tentation incessante de couper la parole est une épée de Damoclès encore plus difficile à repousser, au risque de couper le fil d'autrui pour suivre son propre fil.

La question est de savoir ce que l'on cherche finalement : l'adéquation de souvenirs personnels à des archives (pas seulement audio, d'ailleurs) considérées comme le référent objectif (l'enregistrement audio prenant alors le rôle de l'écrit référent par rapport à l'oral déférent, si l'on peut dire), ou bien encourage-t-on le tissage personnel 
d'une mémoire théâtrale à analyser ensuite, en en privilégiant, pour ce qui occupe le projet $\mathrm{ECHO}$, le registre phonique ? Le chercheur tient en équilibre entre le but de sa recherche et cet objet forcément élusif qu'est la mémoire de son interlocuteur, qui se construit sous ses yeux, ou plutôt ses oreilles, avec lui. Il ne s'agit donc pas de savoir en quoi une archive audio est fidèle à un témoignage, ni l'inverse. Ajoutons que du point de vue de l'efficacité, les éléments factuels ne réactivent pas forcément l'expérience vécue. Les spectateurs qui tiennent des journaux, des carnets de notes de spectacles, qui consultent leurs anciens programmes avant ou pendant l'entretien, ou même des extraits audiovisuels disponibles en ligne, en conviennent eux-mêmes: le souvenir n'est pas toujours ranimé au vu d'une distribution, d'une photo ou d'une autre information factuelle.

19 Le sera-t-il par une écoute rétrospective d'un spectacle déjà vu? L'écoute d'archives audio par des spectateurs, volet prévu dès l'élaboration de cette recherche sur la mémoire théâtrale, n'a pas encore été réalisée pour plusieurs raisons : sa préparation soulève plusieurs questions, tant du point de vue logistique que méthodologique, qui révèlent combien sa pertinence déborde finalement du cadre de recherche initial.

D'abord, même si l'on retrouve le spectacle indiqué par un spectateur dans les archives audio de la BnF, il y a peu de chances que ce soit précisément celui qu'il a vu. Et pourtant l'on sait combien les représentations peuvent différer d'un jour à l'autre, même si l'on met de côté les changements de distribution ou les incidents imprévus. Il sera possible de retrouver la date exacte de la sortie au théâtre, dans certains cas (grâce aux programmes collectés et datés précisément par certaines personnes ou à d'autres documents personnels), mais certainement pas pour leur majorité. Par ailleurs, les conditions d'accès pour l'écoute de ces archives obligeront probablement de demander aux volontaires de se déplacer sur le lieu même des archives, à la BnF, ce qui constitue une contrainte logistique non négligeable. Ensuite, pouvant difficilement écouter une représentation complète, comment sélectionner un extrait si aucun passage précis n'a été évoqué dans les souvenirs?

21 Si l'idée première était d'exposer la mémoire à des retrouvailles dont on attend qu'elles l'éclairent et la fortifient, n'est-ce pas plutôt une nouvelle expérience qui est proposée, qui complexifie la première de plusieurs façons, peut-être même la déstabilise au lieu de la consolider : elle n'est qu'auditive, et enregistrée, au lieu d'être multisensorielle et in vivo, elle ajoute probablement une représentation différente de l'initiale, elle se déroule dans un contexte matériel et humain complètement différent de celui d'une salle de spectacle et d'une sortie au spectacle très souvent faite avec des proches, elle remplace l'initiative du spectateur autonome par sa curiosité et sa collaboration à une démarche de recherche. Finalement, à quels effacements, modifications ou surimpressions expose-t-on la mémoire déjà reconstruite et recréée lors du premier entretien verbal, sans support archivistique ${ }^{13}$ ? Quelles seront les répercussions sur ce premier travail de remémoration? La situation est un entre-deux inédit entre une expérience d'auditeur et la quête d'une mémoire de spectateur de théâtre.

On a vu que la consultation plus importante des archives audio après les entretiens correspond à une étape particulière, quand la moisson de témoignages a pris de l'ampleur, mais aussi quand le chercheur a écouté les enregistrements audio des entretiens qu'il a menés lui-même. La prise de recul nécessaire au dépouillement de ces matériaux originaux et personnels, donc difficiles à analyser, peut alors bénéficier d'une autre écoute, celle d'un matériau dépersonnalisé mais pertinent, les archives 
audio des fonds déposés en bibliothèque. On peut alors attendre un croisement fertile entre les témoignages enregistrés et les archives théâtrales déjà existantes, qui redonnent au chercheur la distance utile à son analyse tout en le maintenant dans le même exercice et la même acuité d'écoute et d'attention. Cette continuité et cette adéquation du médium à son objet, la mémoire phonique, sont à mon avis un atout, et une voie pionnière d'exploration. Mais le chercheur, comme l'amateur de théâtre, doit accepter une contrainte matérielle, celle de l'incompressibilité du temps, de la durée nécessaire à toute écoute, dont il a l'habitude de s'affranchir grâce aux raccourcis de l'écrit et du visuel.

Si l'on considère l'écoute des archives audio comme une expérience à la fois en miniature (c'est-à-dire réduite au son pour un objet conçu à l'origine de manière multisensorielle) et en expansion (cette unique sollicitation sensorielle libérant par contrecoup toute l'attention et l'imaginaire de l'écoutant), on comprend l'intérêt qu'elle représente pour l'analyse d'entretiens eux-mêmes enregistrés, et pour les questions récurrentes que sont la mémoire et l'oubli, les erreurs factuelles, la compression mémorielle dans la durée ou dans la répétition, la mémoire sonore, la relation aux textes, au répertoire, les interactions avec d'autres expériences théâtrales, artistiques ou sociales, ou encore avec des éléments biographiques ${ }^{14}$.

\section{NOTES}

1. Le choix de l'expression "archives audio " plutôt qu' « archives sonores » correspond au fait que les archives filmées ou vidéo, qui peuvent contenir à la fois image et son, n'ont été sollicitées qu'incidemment au cours de cette recherche.

2. Sur cette relation entre quête verbale et quête des souvenirs, et la méthode suivie pour ces entretiens, voir Hélène Bouvier, «Expérience auditive et mémoire phonique dans les entretiens avec des spectateurs de théâtre ", L'Écho du théâtre. Dynamiques et construction de la mémoire phonique, $\mathrm{XX}^{e}$-XXI ${ }^{e}$ siècles, Revue Sciences/Lettres, $\mathrm{n}^{\circ}$ 5, 2017, mis en ligne le 2 octobre 2017, https:// journals.openedition.org/rsl/1034

3. Antonio Damasio résume ainsi le fonctionnement de la mémoire (italiques dans le texte) : « Au lieu d'enregistrer la structure d'une entité, en réalité, le cerveau enregistre les conséquences multiples des interactions de l'organisme avec l'entité concernée. [...] Ce que nous appelons en temps normal le souvenir d'un objet, c'est le souvenir composite des activités sensorielles et motrices liées à l'interaction entre l'organisme et l'objet pendant un certain laps de temps. L'éventail des activités sensorimotrices varie selon la valeur de l'objet et des circonstances. Et son étendue fluctue aussi en fonction d'eux. Nos souvenirs de certains objets sont régis par notre connaissance passée d'objets comparables ou de situations similaires à celle que nous vivons. C'est pourquoi nos souvenirs sont sujets aux préjugés, au sens plein de ce terme, lesquels sont liés à notre histoire passée et à nos croyances. Une mémoire parfaitement fiable est un mythe qui ne vaut que pour des objets triviaux. L'idée selon laquelle le cerveau pourrait avoir un "souvenir de l'objet" isolé ne semble pas tenable. Il garde un souvenir de ce qui s'est passé pendant une interaction, et cette dernière comprend notre passé, ainsi que souvent celui de notre espèce biologique et de notre culture. » L'Autre moi-même. Les nouvelles cartes du cerveau, de la conscience et des émotions, Paris, 
Odile Jacob, 2012 [Self Comes to Mind. Constructing the Conscious Brain, New York, Pantheon Books, 2010], p. 166-167.

4. L'écoute du spectacle, à partir d'un fichier audio sur trois bandes numérisées, s'est faite au casque, en prenant des notes, et après avoir consulté en documentation papier l'ouvrage Jean Vilar par lui-même (Avignon, Maison Jean Vilar, 1991).

5. Ces associations multiples correspondent au processus expliqué ainsi par Antonio Damasio (L'Autre moi-même. Les nouvelles cartes du cerveau, de la conscience et des émotions, op. cit., p. 167) : « Le fait que nous percevions par engagement et non par réceptivité passive est le secret qui explique l'“effet proustien" de la mémoire. C'est pourquoi nous nous souvenons souvent de contextes plutôt que de choses isolées" ".

6. En Indonésie, à Madura, en milieu rural, depuis 1985. Parmi les dernières publications, voir Hélène Bouvier, «Le Loddrok. Entre volontés et contraintes, les métamorphoses d'un genre théâtral », Revue d'histoire du théâtre, vol. IV, n 272, 2016, p. 77-144; " Dynamiques théâtrales en Indonésie : le dernier demi-siècle à Madura ", in Corinne Flicker et al. (dir.), Les Théâtres d'Asie à l'épreuve de la modernité, Paris, Les Indes savantes, à paraître.

7. Les plages durent 2 à 7 minutes, regroupant des enregistrements en public ou en studio des pièces suivantes: Meurtre dans la cathédrale, Le Prince de Hombourg, La Mort de Danton, Nucléa, Lorenzaccio, Richard II, L'Avare, Dom Juan, Le Malade imaginaire, Ruy Blas, Cinna, Macbeth, Henri IV, Ubu, CEdipe, Les Caprices de Marianne, On ne badine pas avec l'amour, La Résistible Ascension d'Arturo Ui, L'Heureux Stratagème, Roses rouges pour moi, L'Alcade de Zalaméa, Les Rustres, La guerre de Troie n'aura pas lieu, Thomas More ou L'homme seul.

8. Disque mono, Hommage à Gérard Philipe, Véga, 1960, 33 t., archives RTF.

9. Le Programme (TNP, L'Arche), vol. 1952-1953, regroupant Le Cid, Mère Courage, Le Prince de Hombourg, L'Avare; le Programme (TNP, L'Arche), vol. 1953-1956, regroupant La Mort de Danton, Richard II, Le Médecin malgré lui, La Garde-Malade, Dom Juan; le Programme (TNP, L'Arche) de Lorenzaccio et le catalogue de l'exposition Lorenzaccio à Avignon.

10. Sur deux cassettes audio, mono, Hachette/L'Encyclopédie sonore, 1974 (reproduisant les disques 33 t. d'origine, Hachette/L'Encyclopédie sonore, 1956).

11. Sur deux disques 33 t. mono, $30 \mathrm{~cm}$, et un disque $33 \mathrm{t}$. mono, $25 \mathrm{~cm}$, Hachette/L'Encyclopédie sonore, 1955.

12. Des incursions sur une période plus récente ont également été faites, avec l'écoute de Tombeau pour 500000 soldats (Pierre Guyotat et Antoine Vitez), enregistré en public en décembre 1981, et Par les villages (Peter Handke et Claude Régy) enregistré en public en décembre 1983.

13. Eric Kandel explique ainsi : "Pour nous tous, la mémoire explicite permet de traverser l'espace et le temps pour invoquer des états émotionnels et des évènements qui se sont évanouis dans le passé mais cependant continuent de vivre dans nos esprits. Pour autant, se remémorer un souvenir de temps en temps - quelle que soit l'importance du souvenir - ne consiste pas seulement à s'arrêter sur une photo d'un album. L'évocation d'un souvenir est un processus actif. On croit savoir que le cerveau ne stocke que le cœur du souvenir. Lors du rappel, ce cœur est alors complexifié et reconstruit, par soustractions, additions, élaborations et distorsions ". À la recherche de la mémoire. Une nouvelle théorie de l'esprit, trad. Marcel Filoche, Paris, Odile Jacob, 2007 [In Search of Memory: The Emergence of a New Science of Mind, New York, W.W. Norton, 2006], p. 288.

14. Que soient ici remerciées chaleureusement toutes les personnes qui ont accepté de partager leur mémoire de spectateurs au cours de nos entretiens, moteurs enthousiastes de cette exploration dans un domaine de recherche passionnant pour toutes les parties mais qui déborde aussi dans un domaine privé souvent imprévisible: en dehors des personnes qui ont souhaité rester anonymes, Isabelle Bloch, Marie-Louise Bloch, Simone Drouin, Geneviève Dubois-Kervran, Françoise Gondal, Lionel Héligoin †, Françoise Hubert-Guillou, Jacques Kraemer, Patrick Liegibel, Pierrette Lombès, Christian Lot, Maud Rayer, Annick Roussel, Jean Roux, Yves Sartiaux, Catherine Sfez, Françoise Viatte, Frédérique Victoire, Jean-Marie Villégier. 


\section{RÉSUMÉS}

L'utilisation des archives audio du théâtre déposées à la BnF a fait partie de la méthodologie utilisée pour explorer la mémoire des spectateurs du théâtre français de l'après-guerre jusqu'aux années 1990. Des entretiens individuels, sans support visuel ni sonore, ont été menés parallèlement. En recourant aux archives audio dans sa préparation, le chercheur favorise-t-il sa rencontre avec un spectateur de théâtre? Quel est l'intérêt particulier de ces archives du théâtre, parmi les autres sources disponibles, écrites et visuelles, pour la compréhension et l'analyse de la mémoire théâtrale d'autrui, et de la place qu'y tiennent le son et l'oralité?

The theatre sound archives in the Bibliothèque nationale de France were used to explore the recollections of French theatregoers from the post-World War II period until the 1990s. The present researcher examined the archives, although interviews with individual spectators were conducted without audio or visual support. Does this prior use of the audio archives help prepare the researcher for such interviews? Among the many visual and textual sources available, what special value do these theatre archives have for understanding and analyzing people's theatrical memory and the traces therein of sound and voice?

\section{INDEX}

Keywords : sound archives, theatrical memory, spectator memory, interviews

Mots-clés : archives audio, mémoire théâtrale, mémoire du spectateur, entretiens

\section{AUTEUR}

\section{HÉLÈNE BOUVIER}

Hélène Bouvier est directeur de recherche au CNRS, anthropologue, HDR en études théâtrales, membre de l'unité mixte de recherche THALIM « Théorie et histoire des arts et des littératures de la modernité XIX ${ }^{\mathrm{e}}-\mathrm{XXI}{ }^{\mathrm{e}}$ siècles » (UMR7172, CNRS, École normale supérieure, université Sorbonne nouvelle-Paris 3 ).

Parmi ses publications :

La Matière des émotions. Les arts du temps et du spectacle dans la société madouraise (Indonésie), Paris, École française d'Extrême-Orient, 1994.

Theatre Research International. Special Issue: Anthropology and Theatre (éd. invité), vol. 19, n 1 , Oxford University Press, 1994.

Théâtres d'Asie à l'œuvre. Circulation, expression, politique, avec G. Toffin (dir.), Paris, École française d'Extrême-Orient, 2012.

L'Art du pathétique en Asie du Sud-Est insulaire. Le choix des larmes (dir.), Paris, L'Harmattan, 2013. L'Écho du théâtre. Dynamiques et construction de la mémoire phonique, $\mathrm{XX}^{e}-\mathrm{XXI} \mathrm{I}^{e}$ siècles, avec $\mathrm{M}$. Chénetier-Alev (dir.), Revue Sciences/Lettres [en ligne], $n^{\circ}$ 5, 2017, Rue d'Ulm, mis en ligne le 2 octobre 2017, https://journals.openedition.org/rsl/1034 